CONFORMAL GEOMETRY AND DYNAMICS

An Electronic Journal of the American Mathematical Society

Volume 1, Pages 1-12 (May 22, 1997)

S $1088-4173(97) 00012-\mathrm{X}$

\title{
THIRTY-THREE YES OR NO QUESTIONS ABOUT MAPPINGS, MEASURES, AND METRICS
}

\author{
JUHA HEINONEN AND STEPHEN SEMMES
}

\section{INTRODUCTION}

Most problems in the ensuing list are of fairly recent origin. None of them seem easy and some are likely to be very difficult. The formulation of each problem is such that it can be answered by one word only: either yes or no. (Strictly speaking, it is conceivable that within the same question, the answer sometimes depends on the dimension.) We offer no conjectures or guesses. In many cases, the particular question is just a chosen concise representative from a whole group of related open problems.

Whenever known, we shall point out the original source of a question. Otherwise, the question is either a folk question, a modification of a folk question, or suggested by one or both of the authors. We apologize in advance for all omissions and misquotes.

Practically all the problems require some background definitions; many concepts that are being used have only recently been introduced, and are perhaps not so widely known. Typically, the question is stated first, and the relevant definitions and references are given right afterwords.

To keep this essay brief, we give little or no motivation here. For this purpose, we kindly invite the reader to consult the literature as referred to in the text.

This list of questions was born at the Institut des Hautes Études Scientifiques in August 1996. The choices we made were necessarily partial but still somewhat arbitrary. There certainly are many more problems around these topics that we deem equally worthy.

\section{The Questions}

Question 1. Is every strong $A_{\infty}$ weight in $\mathbf{R}^{2}$ comparable to the Jacobian of a quasiconformal map $f: \mathbf{R}^{2} \rightarrow \mathbf{R}^{2}$ ?

A strong $A_{\infty}$ weight in $\mathbf{R}^{2}$ is a locally integrable nonnegative function $w$ in $\mathbf{R}^{2}$ so that the induced measure $d \mu=w d \lambda_{2}$ is doubling and that the distance function

$$
D_{w}(x, y)=\left(\int_{B_{x, y}} w d \lambda_{2}\right)^{1 / 2}, \quad x, y \in \mathbf{R}^{2},
$$

Received by the editors February 17, 1997.

1991 Mathematics Subject Classification. Primary 28A75, 30C65; Secondary 53C23, 57M12.

The first author is supported by NSF grant DMS 96-22844. The second author is supported by NSF grant DMS 94-00230. Both authors wish to thank the Institut des Hautes Études Scientifiques for its support. 
is bi-Lipschitz equivalent to a metric in $\mathbf{R}^{2}$; that is, it is asked there be a metric $d_{w}$ in $\mathbf{R}^{2}$ and a constant $C \geq 1$ so that

$$
C^{-1} d_{w}(x, y) \leq D_{w}(x, y) \leq C d_{w}(x, y)
$$

for all pairs of points $x, y \in \mathbf{R}^{2}$. Above, $B_{x, y}$ denotes the (closed) disk in $\mathbf{R}^{2}$ which contains both $x$ and $y$ and has diameter $|x-y|$, and $\lambda_{2}$ denotes the Lebesgue 2-measure.

A nontrivial Borel measure $\mu$ in a metric space $X$ is said to be doubling if there is a constant $C \geq 1$ so that

$$
\mu(2 B) \leq C \mu(B)
$$

for each ball $B$ in $X$, where $2 B$ denotes a ball of same center but twice the radius of $B$.

A weight $w$ is said to be comparable to the Jacobian $J(x, f)$ of a quasiconformal map $f$ if there is a constant $C \geq 1$ so that

$$
C^{-1} J(x, f) \leq w(x) \leq C J(x, f)
$$

for a.e. $x$. An equivalent way to ask Question 1 is to ask whether the metric space $\left(\mathbf{R}^{2}, d_{w}\right)$ is bi-Lipschitz equivalent to $\mathbf{R}^{2}$ with the standard metric, if $w$ is a strong $A_{\infty}$ weight.

Question 1 is due to David and Semmes [DS1]. For more information about strong $A_{\infty}$ weights, see [S2], [S4], [S6, Section 19].

The answer to a similar question in dimensions $n \geq 3$ is no by [S4].

Question 2. Is every $A_{1}$ weight in $\mathbf{R}^{n}, n \geq 2$, comparable to the Jacobian of a quasiconformal map $f: \mathbf{R}^{n} \rightarrow \mathbf{R}^{n}$ ?

An $A_{1}$ weight in $\mathbf{R}^{n}$ is a locally integrable nonnegative function $w$ in $\mathbf{R}^{n}$ for which there exists a constant $C \geq 1$ so that

$$
R^{-n} \int_{B_{R}} w d \lambda_{n} \leq C \operatorname{essinf}_{B_{R}} w
$$

for all $n$-balls $B_{R}$ of radius $R>0$. Here $\lambda_{n}$ is the Lebesgue $n$-measure.

Every $A_{1}$ weight is a strong $A_{\infty}$ weight [S2], so in dimension $n=2$, Question 2 is a special case of Question 1. Question 2 originates in [DS1] as well.

Question 3. If $\left(\mathbf{S}^{2}, d\right)$ is both linearly locally contractible and Ahlfors 2-regular, is it then quasisymmetrically equivalent to $\mathbf{S}^{2}$ ?

Here $\mathbf{S}^{2}$ is the standard 2-sphere and $\left(\mathbf{S}^{2}, d\right)$ denotes a metric space that is homeomorphic to $\mathbf{S}^{2}$.

A metric space is said to be locally linearly contractible if there is a constant $C \geq 1$ so that each metric ball of radius $0<R<C^{-1}$ in the space can be contracted to a point inside a ball of same center but radius $C R$. See [S5] for more discussion about this condition.

A metric space $X$ is said to be Ahlfors s-regular for some real number $s>0$ if it has Hausdorff dimension $s$ and if there is a constant $C \geq 1$ such that

$$
C^{-1} R^{s} \leq \mathcal{H}_{s}\left(B_{R}\right) \leq C R^{s}
$$

for each metric ball $B_{R}$ of radius $R<\operatorname{diam} X$. Here $\mathcal{H}_{s}$ denotes the $s$-dimensional Hausdorff measure in $X$. It is easy to see that a metric space is Ahlfors $s$-regular if $(2.2)$ is satisfied by some Borel measure in place of $\mathcal{H}_{s}$. 
A homeomorphism $f:(X, d) \rightarrow\left(X^{\prime}, d^{\prime}\right)$ between two metric spaces is said to be quasisymmetric if there is a homeomorphism $\eta:[0, \infty) \rightarrow[0, \infty)$ so that

$$
d(x, y) \leq t d(x, z) \quad \text { implies } \quad d^{\prime}(f(x), f(y)) \leq \eta(t) d^{\prime}(f(x), f(z))
$$

for all triples of points $x, y, z$ in $X$. See [TV1] for the basic theory of quasisymmetric maps.

If $d$ is a smooth Riemannian metric on $\mathbf{S}^{2}$, then the answer to Question 3 is yes, in the sense that the quasisymmetry function $\eta$ can be made to depend only on the constants associated with the linear local contractibility and Ahlfors regularity conditions. See [S1], [DS2], [HK2].

The answer to a similar question in dimension $n \geq 3$ is no by [S3]; also the smooth version fails for $n \geq 3$ by [S3].

Question 4. If $\left(\mathbf{S}^{2}, d\right)$ is both linearly locally contractible and Ahlfors 2-regular, is it then bi-Lipschitz equivalent to $\mathbf{S}^{2}$ ?

A homeomorphism $f:(X, d) \rightarrow\left(X^{\prime}, d^{\prime}\right)$ between metric spaces is said to be bi-Lipschitz if there is a constant $C \geq 1$ so that

$$
C^{-1} d(x, y) \leq d^{\prime}(f(x), f(y)) \leq C d(x, y)
$$

for all $x, y \in X$. Bi-Lipschitz maps are always quasisymmetric, so a yes answer to Question 4 implies a yes answer to Question 3. Moreover, a yes answer to Question 4 would imply that the answer to Question 1, and hence to Question 2, is also yes. Next, if the answer to both Questions 1 and 3 is yes, then the answer to Question 4 is also yes.

The answer to a similar question in dimension $n \geq 3$ is false. See [SS] for $n \geq 5$ and [S4] for $n \geq 3$.

Question 5. If $\left(\mathbf{S}^{n}, d\right), n \geq 2$, is linearly locally contractible, Ahlfors n-regular, and quasisymmetrically three point homogeneous, is it then quasisymmetrically equivalent to $\mathbf{S}^{n}$ ?

A metric space $X$ is said to be quasisymmetrically three point homogeneous if for every two triples of distinct points $x, y, z$ and $x^{\prime}, y^{\prime}, z^{\prime}$ in $X$ there is a quasisymmetric self-homeomorphism $f$ of $X$ so that $f(x)=x^{\prime}, f(y)=y^{\prime}$, and $f(z)=z^{\prime}$. Note that both the homogeneity and the linear local contractibility of $\left(\mathbf{S}^{n}, d\right)$ are necessary conditions for the existence of a quasisymmetric map $\left(\mathbf{S}^{n}, d\right) \rightarrow \mathbf{S}^{n}$.

Question 6. Do smooth $n$-dimensional chord arc surfaces with small constant in $\mathbf{R}^{n+1}, n \geq 3$, admit quasisymmetric parametrizations?

A chord arc surface with small constant is defined in [S1, p. 172]. Such a surface $\Gamma$ is assumed smooth, and Question 6 asks if there is an $\eta$-quasisymmetric map $f: \Gamma \rightarrow \mathbf{R}^{n}$ with $\eta$ depending only on the parameters associated with $\Gamma$. The answer to a similar question in dimension $n=2$ is yes by [S1].

Question 7. Do smooth $n$-dimensional chord arc surfaces with small constant in $\mathbf{R}^{n+1}, n \geq 2$, admit bi-Lipschitz parametrizations?

Akin to Question 6, this question asks for the existence of a bi-Lipschitz map $f: \Gamma \rightarrow \mathbf{R}^{n}$ with bi-Lipschitz constant depending only on the parameters associated with the smooth chord arc surface $\Gamma$. Clearly, a yes answer to Question 7 implies a yes answer to Question 6. 
Question 8. If an Ahlfors regular metric space admits a regular map into some Euclidean space, then does it admit a bi-Lipschitz map into another, possibly different, Euclidean space?

A map from one metric space $X$ into another metric space $X^{\prime}$ is said to be regular if it is Lipschitz and if there is a constant $C \geq 1$ so that the preimage of each ball of radius $R$ in $X^{\prime}$ can be covered by at most $C$ balls of radius $R$ in $X$. Recall that a map $f: X \rightarrow X^{\prime}$ is Lipschitz if it satisfies the second inequality in (2.3). Regular maps were first considered by David in [Dd]. For more information about regular maps, see [DS4], [S4].

The works [S4] and [HR1] show that there need not be a bi-Lipschitz map from a metric space $X$ into $\mathbf{R}^{3}$ if there is a regular map from $X$ into $\mathbf{R}^{3}$, even if $X$ is quasisymmetrically homeomorphic to $\mathbf{R}^{3}$.

Question 9. If an Ahlfors n-regular metric space has big pieces of Lipschitz images of $\mathbf{R}^{n}, n \geq 2$, is it then uniformly rectifiable of dimension $n$ ?

A metric space $X$ has big pieces of Lipschitz images of $\mathbf{R}^{n}$ if there exist positive constants $\theta$ and $M$ so that for each $x \in X$ and $0<R<\operatorname{diam} X$ there is a subset $E$ of $\mathbf{R}^{n}$ and an $M$-Lipschitz map $f: E \rightarrow B(x, R)$ so that the Hausdorff $n$-measure of $f(E)$ in $X$ is at least $\theta R^{n}$. Here $B(x, R)$ denotes an open ball in $X$.

An Ahlfors $n$-regular metric space $X$ is said to be uniformly rectifiable of dimension $n$ if there exist positive constants $\theta$ and $M$ so that for each $x \in X$ and $R$ as above there is a subset $A$ of the ball $B(x, R)$ so that the Hausdorff $n$-measure of $A$ is at least $\theta R^{n}$ and that $A$ is $M$-bi-Lipschitz equivalent to a subset of $\mathbf{R}^{n}$.

The answer to Question 9 is yes for Ahlfors regular spaces that are subsets of some Euclidean space by [DS3, Theorem 1.57]. Moreover, positive results for the nonuniform version of Question 9 can be found in [K].

Question 9 is due to David and Semmes [DS4].

Question 10. Does the space $W \times \mathbf{R}^{k}$ admit for some $k \geq 1$ a quasisymmetric map onto $\mathbf{R}^{3+k}$, where $W$ is a linearly locally contractible and Ahlfors 3-regular geometric realization of the decomposition space associated with the Whitehead continuum?

The decomposition space associated with the Whitehead continuum is obtained from $\mathbf{R}^{3}$ by collapsing the Whitehead continuum to a point; see [Dn]. In [S3], it was shown that this space can be realized geometrically as a linearly locally contractible and Ahlfors 3-regular subset of $\mathbf{R}^{4}$, which is a smooth submanifold of $\mathbf{R}^{4}$ save at one point. It is known that $W \times \mathbf{R}$ is homeomorphic to $\mathbf{R}^{4}$; see [Dn, Section 11].

Question 11. Does the space $B \times \mathbf{R}^{k}$ admit for some $k \geq 1$ a quasisymmetric map onto $\mathbf{R}^{3+k}$, where $B$ is a linearly locally contractible and Ahlfors 3-regular geometric realization of the decomposition space associated with the Bing double?

The decomposition space $B$ is described in [Dn], and its pertinent geometric realization in [S3]. This geometric realization is a hypersurface in $\mathbf{R}^{4}$ that is smooth off a Cantor set; it is homeomorphic to $\mathbf{R}^{3}$ but not quasisymmetrically so by [S3].

Question 12. Is there a quasisymmetric map from a polyhedral Edwards sphere $X$ onto $\mathbf{S}^{5}$ ?

This question was raised by Siebenmann and Sullivan in [SS], who also showed that no homeomorphism from $X$ onto $\mathbf{S}^{5}$ (with the standard metric) can be Lipschitz. By a polyhedral Edwards sphere we mean the double suspension of the 
Poincaré homology sphere $H^{3}$, where $H^{3}$ is being triangulated so that the space $X=\Sigma^{2} H^{3}$ as a finite simplicial complex has a natural barycentric metric. Alternatively, we can embed $X$ piecewise linearly into some Euclidean space and take the induced Euclidean metric. For this problem, it does not matter which underlying triangulation one chooses for $H^{3}$.

Due to deep work of Edwards and Cannon, double suspensions of homology spheres are known to be homeomorphic to standard spheres; see [C], [E], and [Dn]. No explicit modulus of continuity of such a homeomorphism is known to the authors. Note that a quasisymmetric map from $X$ onto $\mathbf{S}^{5}$ would be Hölder continuous.

The next two questions ask further how good a homeomorphism from $X$ to $\mathbf{S}^{5}$ can be.

Question 13. Is there a homeomorphism from a polyhedral Edwards sphere X onto $\mathbf{S}^{5}$ that preserves sets of Hausdorff 5-measure zero?

We have a natural 5-dimensional Hausdorff measure on $X$, which restricts to the Lebesgue 5-measure on each 5-simplex. By [HK3, Section 7], a yes answer to Question 12 implies a yes answer to Question 13.

Question 14. Is there a homeomorphism from a polyhedral Edwards sphere $X$ onto $\mathbf{S}^{5}$ that belongs to the Sobolev space $W^{1, p}(X)$ for some $p \geq 1$ ?

We say that a homeomorphism $f: X \rightarrow \mathbf{S}^{5}$ belongs to the Sobolev space $W^{1, p}(X)$ if it belongs to the standard Sobolev space $W^{1, p}\left(\Delta_{5}\right)$ on each 5 -simplex $\Delta_{5}$ on $X$ as a map $\Delta_{5} \rightarrow \mathbf{S}^{5} \subset \mathbf{R}^{6}$.

Question 15. If $X$ is a metric space of locally finite Hausdorff $n$-measure and $f$ a quasisymmetric homeomorphism from $X$ onto $\mathbf{R}^{n}, n \geq 2$, is $f$ then absolutely continuous with respect to the Hausdorff n-measures?

By absolute continuity of $f$ we mean that $f$ carries sets of Hausdorff $n$-measure zero in $X$ to sets of Hausdorff (Lebesgue) $n$-measure zero in $\mathbf{R}^{n}$. This question appears in a paper by Väisälä [V2, Question 5.10] for subsets $X$ of Euclidean space, and the answer is unknown even in that case. The answer is known to be yes if $X$ is Ahlfors $n$-regular [DS1], [H1], [HK3]. It is well known that the answer to a similar question is $n o$ for $n=1$.

If $f$ is a quasisymmetric embedding of $\mathbf{R}^{n}, n \geq 2$, into some Euclidean space so that the image $f\left(\mathbf{R}^{n}\right)$ has locally finite Hausdorff $n$-measure, then $f$ is absolutely continuous in that it carries sets of $n$-measure zero to sets of $n$-measure zero; this is due to Väisälä [V2] who extended the earlier result of Gehring [G1], [G2] from the case where $f$ has a quasisymmetric extension to $\mathbf{R}^{N}$, where $N \geq n$ is such that $f\left(\mathbf{R}^{n}\right) \subset \mathbf{R}^{N}$.

Question 16. If $X$ is a metric space and $f$ a quasisymmetric homeomorphism from $X$ onto $\mathbf{R}^{n}, n \geq 2$, is $f$ then absolutely continuous with respect to the Hausdorff $n$-measures?

Question 16 asks if $f$ carries sets of Hausdorff $n$-measure zero in $X$ to sets of Hausdorff (Lebesgue) $n$-measure zero in $\mathbf{R}^{n}$. The only difference to Question 15 is that no assumption on the measure properties of $X$ is being made. However, since $X$ is homeomorphic to $\mathbf{R}^{n}$, its Hausdorff dimension is at least $n$ by the dimension theory [HW, Chapter VII]. 
Clearly, a yes answer to Question 16 implies a yes answer to Question 15. Again, the answer is unknown even for subsets $X$ of Euclidean space, and the answer to a similar question is $n o$ if $n=1$.

See [H2], [HK1], [S8], [V3] for further discussion and problems along these lines.

Question 17. Is there an embedding $f$ of $\mathbf{R}^{2}$ into $\mathbf{R}^{3}$ such that, for some $C \geq 1$ and $0<\alpha<1$,

$$
C^{-1}|x-y|^{\alpha} \leq|f(x)-f(y)| \leq C|x-y|^{\alpha}
$$

for all $x, y \in \mathbf{R}^{2}$ ?

The answer to a similar question where 2 and 3 are replaced by 1 and 2, respectively, is yes, as demonstrated by the standard von Koch snowflake. See [F, p. 121]. The answer is likewise yes if one allows the image to lie in a larger dimensional Euclidean space; see $[\mathrm{A}]$. Note that an embedding $f$ as in (2.4) is quasisymmetric, and necessarily $\alpha>2 / 3$.

Question 18. Is there a quasisymmetric embedding $f$ of $\mathbf{R}^{2}$ into $\mathbf{R}^{3}$ such that the image $f\left(\mathbf{R}^{2}\right)$ contains no rectifiable curves?

This question is due to Steffen Rohde. Clearly, if the answer to Question 17 is yes, the answer to Question 18 is yes as well.

Question 19. If $X$ is an Ahlfors $Q$-regular space that admits a weak $(1,1)$-Poincaré inequality, is $Q$ then an integer?

An Ahlfors $Q$-regular space is said to satisfy a weak (1,1)-Poincaré inequality, if there are constants $C_{1} \geq 1$ and $C_{2} \geq 1$ so that

$$
\int_{B_{R}}\left|u-u_{B}\right| d \mu \leq C_{1} R \int_{B_{C_{2} R}} \rho d \mu
$$

for all balls $B_{R}$ of radius $0<R<\operatorname{diam} X$, for all continuous functions $u$ defined in $B_{C_{2} R}$, and for all upper gradients $\rho$ of $u$ in $B_{C_{2} R}$. A nonnegative Borel function $\rho$ is said to be an upper gradient of a function $u$ defined on a metric space if

$$
|u(x)-u(y)| \leq \int_{\gamma} \rho d s
$$

for all points $x$ and $y$ and for all rectifiable curves $\gamma$ joining the two points.

See [HK3] and [S5] for more about Poincaré inequalities and upper gradients (called very weak gradients in [HK3]).

Carnot groups are examples of $Q$-regular spaces that are topological manifolds of dimension less than $Q$ but yet admit a weak $(1,1)$-Poincaré inequality; see [J].

Question 20. If $X$ is an Ahlfors $Q$-regular Loewner space for some $Q>1$, is $Q$ then an integer?

An Ahlfors $Q$-regular metric space $X$ is said to be a Loewner space if the function

$$
\lambda(t)=\inf \{\bmod (E, F ; X): \Delta(E, F) \leq t\}
$$


is positive for all $t>0$, where $E$ and $F$ are disjoint nondegenerate continua in $X$,

and

$$
\Delta(E, F)=\frac{\operatorname{dist}(E, F)}{\min \{\operatorname{diam} E, \operatorname{diam} F\}},
$$

$$
\bmod (E, F ; X)=\inf \int_{X} \rho^{Q} d \mu
$$

with the infimum taken over all nonnegative Borel functions $\rho$ such that

$$
\int_{\gamma} \rho d s \geq 1
$$

for all rectifiable $\gamma$ joining $E$ and $F$. Above, $d \mu$ denotes the Hausdorff $Q$-measure in $X$.

For a more thorough discussion on Loewner spaces, see [HK3].

It was shown in [HK3] that if a $Q$-regular space $X$ admits a weak $(1,1)$-Poincaré inequality, then $X$ is a Loewner space, provided $X$ also satisfies some additional qualitative assumptions (for instance, it suffices to assume that closed balls in $X$ are compact and that $X$ is locally quasiconvex). In particular, Carnot groups are $Q$ regular Loewner spaces with $Q$ larger than the topological dimension. Furthermore, by [HK3, 6.19], there are $Q$-regular Loewner spaces that have different topological dimension at different places.

Question 21. If the first Heisenberg group is deformed by a metric doubling measure, is a (1,1)-Poincaré inequality retained?

The Heisenberg group is assumed equipped with its Carnot metric; thus it is an Ahlfors 4-regular space. For a discussion of the Heisenberg group and the definition of a Carnot metric, see [Pa], [KR1], [KR2].

Recall the definition for a doubling measure from (2.1).

Given a doubling measure $\mu$ in an Ahlfors $Q$-regular space $(X, d)$, we can associate to it a distance function much as in the definition for a strong $A_{\infty}$ weight:

$$
D_{\mu}(x, y)=\mu\left(B_{x, y}\right)^{1 / Q},
$$

where $B_{x, y}=B(x, d(x, y)) \cup B(y, d(x, y))$ is the union of two (closed) balls centered at the points $x$ and $y$, and both with radius $d(x, y)$. The distance function $D_{\mu}$ need not satisfy the triangle inequality, although it satisfies the other requirements for a metric. A doubling measure $\mu$ is called a metric doubling measure if there is a metric $d_{\mu}$ on $X$ which is bi-Lipschitz equivalent to $D_{\mu}$; that is, if there is a constant $C \geq 1$ such that

$$
C^{-1} d_{\mu}(x, y) \leq D_{\mu}(x, y) \leq C d_{\mu}(x, y)
$$

for all $x, y \in X$.

It is not hard to see that the space $\left(X, d_{\mu}\right)$ is also Ahlfors $Q$-regular, and it is called a deformation of $X$ by a metric doubling measure $\mu$. It is also not hard to see that the identity map $(X, d) \rightarrow\left(X, d_{\mu}\right)$ is quasisymmetric.

The answer to Question 21 is yes if the Heisenberg group is replaced with $\mathbf{R}^{n}$. See [DS1], [S5], [S7]. Moreover, in general the answer is no for a $Q$-regular space admitting a weak $(1,1)$-Poincaré inequality; that is, a deformation with a metric doubling measure may destroy a weak $(1,1)$-Poincaré inequality on a general $Q$ regular space by $[\mathrm{HK} 3,6.19]$. However, a somewhat weaker Poincaré inequality is always retained by $[\mathrm{KM}]$. 
Question 22. Is the first Heisenberg group minimal in looking down?

Again, it is understood here that the Heisenberg group is equipped with its Carnot metric.

Question 22 is due to David and Semmes [DS4]. "Looking down" is a partial ordering in the collection of equivalence classes of BPI spaces, where letters BPI stand for "big pieces of itself".

An Ahlfors $Q$-regular metric space $X$ is said to be a BPI space if there are constants $C \geq 1$ and $\theta>0$ so that for each pair of balls $B\left(x_{1}, r_{1}\right)$ and $B\left(x_{2}, r_{2}\right)$ in $X$ with $0<r_{1}, r_{2}<\operatorname{diam} X$ there is a closed set $A \subset B\left(x_{1}, r_{1}\right)$ whose $Q$-Hausdorff measure is at least $\theta r_{1}^{Q}$ and there is a $C$-conformally bi-Lipschitz embedding $f$ : $A \rightarrow B\left(x_{2}, r_{2}\right)$ with scale factor $r_{2} / r_{1}$.

A bi-Lipschitz map $f$ between metric spaces $(X, d)$ and $\left(X^{\prime}, d^{\prime}\right)$ is said to be $C$-conformally bi-Lipschitz with scale factor $\lambda>0$ if $f$ is $C$-bi-Lipschitz between the metric spaces $(X, \lambda d)$ and $\left(X^{\prime}, d^{\prime}\right)$.

Two BPI spaces $X$ and $X^{\prime}$ of the same dimension $Q$ are said to be BPI equivalent if there are positive constants $\theta$ and $M$ so that for each $x \in X, x^{\prime} \in X^{\prime}$ and radii $0<R<\operatorname{diam} X, 0<R^{\prime}<\operatorname{diam} X^{\prime}$ there exist a subset $A$ of the ball $B(x, R)$ in $X$ with $Q$-Hausdorff measure at least $\theta R^{Q}$ and an $M$-conformally bi-Lipschitz embedding $f: A \rightarrow B\left(x^{\prime}, R^{\prime}\right)$ with scale factor $R^{\prime} / R$. It is not hard to see that two BPI spaces of the same dimension $Q$ are BPI equivalent if they have subsets of positive $Q$-measure that are bi-Lipschitz equivalent.

A BPI metric space $X$ of Hausdorff dimension $Q$ is said to look down on another BPI metric space $Y$, also of Hausdorff dimension $Q$, if there is a closed set $A$ in $X$ and a Lipschitz map $f: A \rightarrow Y$ so that $f(A)$ has positive Hausdorff $Q$-measure. If also $Y$ looks down on $X$, then $X$ and $Y$ are look-down equivalent.

A BPI space $X$ of dimension $Q$ is said to be minimal in looking down if every BPI space $Y$ of same dimension $Q$ that $X$ looks down to is BPI equivalent to $X$.

Each Euclidean space is minimal in looking down by [DS4].

Question 23. If two Q-regular BPI metric spaces are look-down equivalent, are they then BPI equivalent?

Question 23 appears in [DS4], where more discussion and more similar problems can be found.

Question 24. If $f$ is a Lipschitz map of a subset $E$ in the first Heisenberg group equipped with its Carnot metric into a metric space such that the Hausdorff 4measure of $f(E)$ is positive, is $f$ then bi-Lipschitz in a subset of $E$ of positive Hausdorff 4-measure?

If the answer to Question 24 is yes, then the answer to Question 22 is yes as well. The answer to a similar question where the Heisenberg group is replaced with $\mathbf{R}^{n}$ is yes by $[\mathrm{K}]$.

Question 24 is again from [DS4].

Question 25. Can the T-axis in the first Heisenberg group be mapped onto a locally rectifiable curve by a quasiconformal self-map of the group?

For a discussion about quasiconformal maps in the Heisenberg group with its Carnot metric, see [KR2]. The definition for quasiconformal maps is taken to be the metric one, where no smoothness is assumed. In fact, quasiconformal self-maps of the Heisenberg group are the same as quasisymmetric maps by [KR2], [HK2]. 
Question 26. Is the fixed point set of every quasiconformal reflection $\mathbf{S}^{3} \rightarrow \mathbf{S}^{3}$ a topologically tame 2-sphere?

A quasiconformal reflection on the 3 -sphere $\mathbf{S}^{3}$ is a sense-reversing quasiconformal involution. Question 26 was raised by Heinonen and Yang [HY]. Note that by a result of Tukia and Väisälä [TV2], one can assume that the reflection is in fact bi-Lipschitz.

Question 27. Can every branched cover $\mathbf{S}^{n} \rightarrow \mathbf{S}^{n}, n \geq 3$, be made BLD by changing the metric in the domain but keeping the space n-regular and linearly locally contractible?

We define a branched cover between manifolds to be a discrete, open, and sensepreserving map. Note in particular that no local tameness assumption is made on the branching locus.

A branched cover $f: X \rightarrow Y$ between metric manifolds is said to be $B L D$, or a map of bounded length distortion, if there is a constant $L \geq 1$ so that

$$
L^{-1} \text { length } \gamma \leq \operatorname{length}(f \circ \gamma) \leq L \text { length } \gamma
$$

for all curves $\gamma$ in $X$. Question 27 asks if for a given branched cover $f: \mathbf{S}^{n} \rightarrow \mathbf{S}^{n}$ there is a metric $d$ on $\mathbf{S}^{n}$ so that $\left(\mathbf{S}^{n}, d\right)$ is an Ahlfors $n$-regular and locally linearly contractible metric space, and $f:\left(\mathbf{S}^{n}, d\right) \rightarrow \mathbf{S}^{n}$ is a BLD map.

The answer to a similar question for $n=2$ is yes; then, in fact, every branched cover is topologically conjugate to a holomorphic map by a classical theorem of Stoïlow.

Question 27 is due to Heinonen and Rickman [HR2]. See [MV], [HR2] for more information about BLD branched covers.

Question 28. Is every branched cover $f: \mathbf{S}^{n} \rightarrow \mathbf{S}^{n}, n \geq 3$, topologically conjugate to a quasiregular map?

Question 28 (in a slightly different local formulation) was stated by Martio, Rickman, and Väisälä in [MRV, 4.11]. For definitions and discussion of quasiregular maps, see the monographs [Re] and [Ri2]. Question 28 asks if for a given branched cover $f$ one can find homeomorphisms $h$ and $g$ of the $n$-sphere such that $h \circ f \circ g$ is quasiregular. Recall that every nonconstant quasiregular map is a branched cover as defined above.

The answer to a similar question in dimension $n=2$ is yes by Stoillow's theorem.

Question 29. Is the topological dimension of the branch set of a quasiregular map $\mathbf{S}^{n} \rightarrow \mathbf{S}^{n}, n \geq 3$, either $-1, n-2$, or $n$ ?

The branch set of a quasiregular map is the closed set of points in the domain where the map does not define a local homeomorphism. Thus, for a quasiregular map $\mathbf{S}^{n} \rightarrow \mathbf{S}^{n}$, the dimension of the branch set is -1 if and only if the map is homeomorphic and it is $n$ if and only if the map is constant. By a result of Chernavskiı̌ [C1], [C2] (see also Väisälä [V1]), the topological dimension of the branched set for any branched cover between $n$-manifolds is at most $n-2$; in dimension $n=2$ it is known to be either -1 or $n-2$ by the aforementioned Stoïlow theorem.

The Edwards sphere can be used to show that there are branched covers $\mathbf{S}^{n} \rightarrow \mathbf{S}^{n}$ for $n \geq 5$ whose branch set is presicely an $(n-4)$-dimensional sphere. Thus, if the answer to Question 29 is yes, then the answer to Questions 11 and 28 is no, and if the answer to either Question 11 or Question 28 is yes, then the answer to Question 29 is no. 
Question 29 has been around since the inception of the theory of quasiregular maps. See $[\mathrm{Sr}]$ for a recent survey and references.

Question 30. Does there exist a nonconstant quasiregular map $\mathbf{R}^{4} \rightarrow \mathbf{S}^{2} \times \mathbf{S}^{2} \sharp \mathbf{S}^{2} \times$ $\mathrm{S}^{2}$ ?

This question is due to Mikhail Gromov and Seppo Rickman. Here $\sharp$ denotes connected sum. It is not difficult to construct a nonconstant quasiregular map $\mathbf{R}^{4} \rightarrow \mathbf{S}^{2} \times \mathbf{S}^{2}$. See [Ri1].

Question 31. Does every closed oriented topological $n$-manifold, $n \geq 4$, admit a branched cover onto $\mathbf{S}^{n}$ ?

By an old theorem of Alexander, the answer is yes to every combinatorially triangulable (closed) manifold; in particular the answer is yes in dimensions $n=1,2,3$. In fact, Alexander's method only requires that the manifold be homeomorphic to a simplicial complex which is an oriented pseudo-manifold (without boundary) as defined in [Sp, p. 148]. It is known that not every closed four-manifold can so be triangulated (see page xvi in the Introduction of $[\mathrm{AM}]$ ), but the existence of a triangulation is unknown in dimensions five or more, cf. [GS]. See [Ri3] for a discussion about Alexander's method.

We reiterate that in the above questions branched covers are not required to be piecewise linear, or even locally equivalent to such. (See the definition after Question 27.)

Question 32. Does every closed oriented $P L$ n-manifold, $n \geq 5$, admit a $P L$ branched cover of degree at most $n$ onto the PL n-sphere?

We learned Question 32 from Dennis Sullivan. The answer to a similar question is yes in dimensions $n=1,2,3,4$. The case $n=2$ is classical, the case $n=3$ was proved independently in the 1970's by Hilden, Hirsch, and Montesinos (see [BE] for an extensive account), while the case $n=4$ is a recent result of Piergallini [Pi]

Question 33. Does every closed topological four-manifold admit a metric that makes the manifold Ahlfors 4-regular and locally linearly contractible?

The answer to a similar question in dimensions other than four is yes, because of Sullivan's theorem on the existence of Lipschitz structures [Su]. In contrast to this, recall that in dimension four most manifolds do not admit smooth or even Lipschitz or quasiconformal structures, by the works of Freedman, Donaldson, and Donaldson and Sullivan; see [FQ], [DK], [DS]. Furthermore, as mentioned above after Question 31, there are four manifolds that are not homeomorphic to finite simplicial complexes.

\section{REFERENCES}

[AM] S. Akbulut and J. D. McCarthy, Casson's invariant for oriented homology 3-spheres: An exposition, vol. 36, Princeton University Press, Mathematical Notes, Princeton, New Jersey, 1990. MR 90k:57017

[A] P. Assouad, Plongements Lipschitziens dans $\mathbf{R}^{n}$, Bull. Soc. Math. France 111 (1983), 429-448. MR 86f:54050

[BE] I. Berstein and A. L. Edmonds, On the construction of branched coverings of lowdimensional manifolds, Trans. Amer. Math. Soc. 247 (1979), 87-124. MR 80b:57003

[C] J. Cannon, The recognition problem: What is a topological manifold?, Bull. Amer. Math. Soc. 84 (1978), 832-866. MR 58:13043

[C1] A. V. Čhernavskiǔ, Finite-to-one open mappings of manifolds (in Russian), Mat. Sb. 65 (1964), 357-369. MR 30:2476 
[C2] A. V. Čhernavskiı̌, Remarks on the paper "Finite-to-one open mappings of manifolds" (in Russian), Mat. Sb. 66 (1965), 471-472. MR 36:3320

[Dn] R. Daverman, Decompositions of manifolds, Academic Press, 1986. MR 88a:57001

[Dd] G. David, Morceaux de graphes lipschitziennes et intégrales singulières sur un surface, Rev. Mat. Iberoamericana 4 (1988), 73-114. MR 90h:42026

[DS1] G. David and S. Semmes, Strong $A_{\infty}$-weights, Sobolev inequalities, and quasiconformal mappings, Analysis and partial differential equations, Lecture Notes in Pure and Applied Mathematics, Marcel Dekker 122 (1990). MR 91c:30037

[DS2] G. David and S. Semmes, Quantitative rectifiability and Lipschitz mappings, Trans. Amer. Math. Soc. 337 (1993), 855-889. MR 93h:42015

[DS3] G. David and S. Semmes, Analysis of and on uniformly rectifiable sets, vol. 38, Amer. Math. Soc., Mathematical Surveys and Monographs, 1993. MR 94i:28003

[DS4] G. David and S. Semmes, Fractured fractals and broken dreams: Self-similarity through metric and measure, Oxford University Press, to appear.

[DK] S. K. Donaldson and P. B. Kronheimer, The geometry of four-manifolds, Oxford University Press, 1990. MR 92a:57036

[DS] S. K. Donaldson and D. P. Sullivan, Quasiconformal 4-manifolds, Acta Math. 163 (1989), 181-252. MR 91d:57012

[E] E. Edwards, The topology of manifolds and cell-like maps, Proceedings ICM, Helsinki (1978), 111-127. MR 81g:57010

[F] K. J. Falconer, Fractal Geometry, John Wiley \& Sons, 1990. MR 92j:28008

[FQ] M. H. Freedman and F. Quinn, Topology of 4-manifolds, vol. 39, Princeton University Press, Princeton Mathematical Series, Princeton, New Jersey, 1990. MR 94b:57021

[GS] D. E. Galewski and R. J. Stern, Classification of simplicial triangulations of topological manifolds, Ann. of Math. 111 (1980), 1-34. MR 81f:57012

[G1] F. W. Gehring, The Hausdorff measure of sets which link in Euclidean space, Contributions to Analysis: A Collection of Papers Dedicated to Lipman Bers, Academic Press, New York, 1974. MR 50:13455

[G2] F. W. Gehring, Lower dimensional absolute continuity properties of quasiconformal mappings, Math. Proc. Camb. Phil. Soc. 78 (1975), 81-93. MR 52:3520

[H1] J. Heinonen, The boundary absolute continuity of quasiconformal mappings, Amer. J. Math. 116 (1994), 1545-1567. MR 96m:30033

[H2] J. Heinonen, A theorem of Semmes and the boundary absolute continuity in all dimensions, Rev. Mat. Iberoamericana 12 (1996), 783-789.

[HK1] J. Heinonen and P. Koskela, The boundary distortion of a quasiconformal mapping, Pacific J. Math. 165 (1994), 93-114. MR 95f:30031

[HK2] J. Heinonen and P. Koskela, Definitions of quasiconformality, Invent. Math. 120 (1995), 61-79. MR 96e:30051

[HK3] J. Heinonen and P. Koskela, Quasiconformal maps in metric spaces with controlled geometry, Preprint, University of Jyväskylä (1996).

[HR1] J. Heinonen and S. Rickman, Quasiregular maps $\mathbf{S}^{3} \rightarrow \mathbf{S}^{3}$ with wild branch sets, Topology (to appear).

[HR2] J. Heinonen and S. Rickman, Geometric branched covers between generalized manifolds, in preparation.

[HY] J. Heinonen and S. Yang, Strongly uniform domains and periodic quasiconformal maps, Ann. Acad. Sci. Fenn. Ser. A. I. Math. 20 (1995), 123-148. MR 96d:30023

[HW] W. Hurewicz and H. Wallman, Dimension Theory, Princeton Univ. Press, Princeton, New Jersey, 1948. MR 3:312b

[J] D. Jerison, The Poincaré inequality for vector fields satisfying Hörmander's condition, Duke Math. J. 53 (1986), 503-523. MR 87i:35027

[K] B. Kirchheim, Rectifiable metric spaces: Local structure and regularity of the Hausdorff measure, Proc. Amer. Math. Soc. 121 (1994), 113-123. MR 94g:28013

[KM] P. Koskela and P. MacManus, Quasiconformal mappings and Sobolev spaces, Preprint (1997).

[KR1] A. Korányi and H. M. Reimann, Quasiconformal mappings on the Heisenberg group, Invent. Math. 80 (1985), 309-338. MR 86m:32035

[KR2] A. Korányi and H. M. Reimann, Foundations for the theory of quasiconformal mappings on the Heisenberg group, Adv. Math. 111 (1995), 1-87. MR 96c:30021 
[MRV] O. Martio, S. Rickman, and J. Väisälä, Topological and metric properties of quasiregular mappings, Ann. Acad. Sci. Fenn. Ser. A I Math. 488 (1971), 1-31. MR 45:8830

[MV] O. Martio and J. Väisälä, Elliptic equations and maps of bounded length distortion, Math. Ann. 282 (1988), 423-443. MR 89m:35062

[Pa] P. Pansu, Métriques de Carnot-Carathéodory et quasiisométries des espaces symétriques de rang un, Ann. Math. 129 (1989), 1-60. MR 90e:53058

[Pi] R. Piergallini, Four-manifolds as 4-fold branched covers of $\mathbf{S}^{4}$, Topology 34 (1995), 497508. MR 96g:57003

[Re] Yu. G. Reshetnyak, Space mappings with bounded distortion, Translation of Mathematical Monographs 73, American Mathematical Society, Providence, RI, 1989. MR 90d:30067

[Ri1] S. Rickman, Existence of quasiregular mappings, Proceedings of the Workshop on Holomorphic Functions and Moduli I. Math. Sci. Res. Inst. Publ. Berkeley, Springer-Verlag 10 (1988), 179-185. MR 90g:30026

[Ri2] S. Rickman, Quasiregular mappings, Ergebnisse der Mathematik und ihrer Grenzgebiete 26, Springer-Verlag, Berlin Heidelberg New York, 1993. MR 95g:30026

[Ri3] S. Rickman, Construction of quasiregular mappings, In the volume in honor of F. Gehring, Springer-Verlag (to appear).

[S1] S. Semmes, Chord-arc surfaces with small constant. II. Good parameterizations, Adv. Math. 88 (1991), 170-199. MR 93d:42019b

[S2] S. Semmes, Bi-Lipschitz mappings and strong $A_{\infty}$ weights, Ann. Acad. Sci. Fenn. Ser. A I Math. 18 (1993), 211-248. MR 95g:30032

[S3] S. Semmes, Good metric spaces without good parameterizations, Rev. Mat. Iberoamericana 12 (1996), 187-275. CMP 96:12

[S4] S. Semmes, On the nonexistence of bilipschitz parameterizations and geometric problems about $A_{\infty}$ weights, Rev. Mat. Iberoamericana 12 (1996), 337-410. CMP 96:16

[S5] S. Semmes, Finding Curves on General Spaces through Quantitative Topology with Applications for Sobolev and Poincaré inequalities, Selecta Math. (N.S.) 2 (1996), 155-295. CMP 97:03

[S6] S. Semmes, Metric structures in Riemannian spaces, M. Gromov et. al., Birkhäuser (to appear).

[S7] S. Semmes, Some remarks about metric spaces, spherical mappings, functions and their derivatives, Publicacions Matemàtiques 40 (1996), no. 2, 411-430. CMP 97:06

[S8] S. Semmes, Quasisymmetry, measure, and a question of Heinonen, Rev. Mat. Iberoamericana 12 (1996), 727-781.

[SS] L. Siebenmann and D. Sullivan, On complexes that are Lipschitz manifolds, Geometric topology (Proceedings Georgia Topology Conf., Athens, Ga. 1977) edited by J. C. Cantrell, Academic Press, New York, N.Y. - London (1979), 503-525. MR 80h:57027

[Sp] E. H. Spanier, Algebraic Topology, McGraw-Hill, New York, 1966. MR 35:1007

[Sr] U. Srebro, Topological properties of quasiregular maps, In Quasiconformal space mappings; a collection of surveys 1960-1990, M. Vuorinen Ed. Springer Verlag, Lecture Notes 1508 (1992), 104-118. CMP 93:02

[Su] D. P. Sullivan, Hyperbolic geometry and homeomorphisms, Geometric topology (Proceedings Georgia Topology Conf., Athens, Ga. 1977) edited by J. C. Cantrell, Academic Press, New York, N.Y. - London (1979), 543-555. MR 81 m:57012

[TV1] P. Tukia and J. Väisälä, Quasisymmetric embeddings of metric spaces, Ann. Acad. Sci. Fenn. Ser. A I Math. 5 (1980), 97-114. MR 82g:30038

[TV2] P. Tukia and J. Väisälä, Bi-Lipschitz extensions of maps having quasiconformal extensions, Math. Ann. 269 (1984), 561-572. MR 86c:30041

[V1] J. Väisälä, Discrete open mappings on manifolds, Ann. Acad. Sci. Fenn. Ser. A I 392 (1966), 1-10. MR 34:814

[V2] J. Väisälä, Quasi-symmetric embeddings in euclidean spaces, Trans. Amer. Math. Soc. 264 (1981), 191-204. MR 82i:30031

[V3] J. Väisälä, The wall conjecture on domains in Euclidean spaces, Manuscripta Math. (to appear).

Department of Mathematics, University of Michigan, Ann Arbor, Michigan 48109

E-mail address: juha.heinonen@umich.edu

Department of Mathematics, Rice University, Houston, Texas 77251

E-mail address: semmes@math.rice.edu 